Mission Statement-IUPAC is a non-governmental organization of member countries that encompass more than $85 \%$ of the world's chemical sciences and industries. IUPAC addresses international issues in the chemical sciences utilizing expert volunteers from its member countries. IUPAC provides leadership, facilitation, and encouragement of chemistry and promotes the norms, values, standards, and ethics of science and the free exchange of scientific information. Scientists have unimpeded access to IUPAC activities and reports. In fulfilling this mission, IUPAC effectively contributes to the worldwide understanding and application of the chemical sciences, to the betterment of the human condition.

President: NICOLE MOREAU (France)

Vice President: KAZUYUKI TATSUMI (Japan)

Past President: JUNG-IL JIN (Korea)
Secretary General: DAVID StC. BLACK (Australia)

Treasurer: JOHN CORISH (Ireland)

\title{
National Adhering Organizations
}

Australian Academy of Science (Australia)

Österreichische Akademie der Wissenschaften (Austria)

Bangladesh Chemical Society (Bangladesh)

The Royal Academies for the Sciences and

Arts of Belgium (Belgium)

Brazilian Chemistry Committee for IUPAC (Brazil)

Bulgarian Academy of Sciences (Bulgaria)

National Research Council of Canada (Canada)

Sociedad Chilena de Química (Chile)

Chinese Chemical Society (China)

Chemical Society located in Taipei (China)

Croatian Chemical Society (Croatia)

Sociedad Cubana de Química (Cuba)

Pancyprian Union of Chemists (Cyprus)

Czech National Committee for Chemistry (Czech Republic)

Det Kongelige Danske Videnskabernes Selskab (Denmark)

National Committee for IUPAC (Egypt)

Chemical Society of Ethiopia (Ethiopia)

Suomen Kemian Seura-Kemiska Sällskapet i

Finland (Finland)

Comité National Français de la Chimie (France)

Deutscher Zentralausschuss für Chemie

(Germany)

Association of Greek Chemists (Greece)

Hungarian Academy of Sciences (Hungary)

Indian National Science Academy (India)

Royal Irish Academy (Ireland)

Israel Academy of Sciences and Humanities (Israel)

Consiglio Nazionale delle Ricerche (Italy)

Caribbean Academy of Sciences-Jamaica

Chapter (Jamaica)
Science Council of Japan (Japan)

Jordanian Chemical Society (Jordan)

Korean Federation of Science and Technology

Societies (Korea)

Kuwait Chemical Society (Kuwait)

Fonds National de la Recherche (Luxembourg)

Institut Kimia Malaysia (Malaysia)

Koninklijke Nederlandse Chemische Vereniging (Netherlands)

Royal Society of New Zealand (New Zealand)

Norsk Kjemisk Selskap (Norway)

Chemical Society of Pakistan (Pakistan)

Polska Akademia Nauk (Poland)

Sociedade Portuguesa de Química (Portugal)

Colegio de Químicos de Puerto Rico

(Puerto Rico)

Russian Academy of Sciences (Russia)

Serbian Chemical Society (Serbia)

Slovak Chemical Society (Slovakia)

Slovenian Chemical Society (Slovenia)

National Research Foundation (South Africa)

Ministerio de Educación y Ciencia (Spain)

Institute of Chemistry, Ceylon (Sri Lanka)

Svenska Nationalkommittén för Kemi (Sweden)

Swiss Chemical Society (Switzerland)

Tanzania Chemical Society (Tanzania)

Chemical Society of Thailand (Thailand)

Société Chimique de Tunisie (Tunisia)

Türkiye Kimya Dernegi (Turkey)

National Academy of Sciences of Ukraine

(Ukraine)

Royal Society of Chemistry (United Kingdom)

National Academy of Sciences (USA)

Programa de Desarrollo de Ciencias Básicas

(Uruguay) 


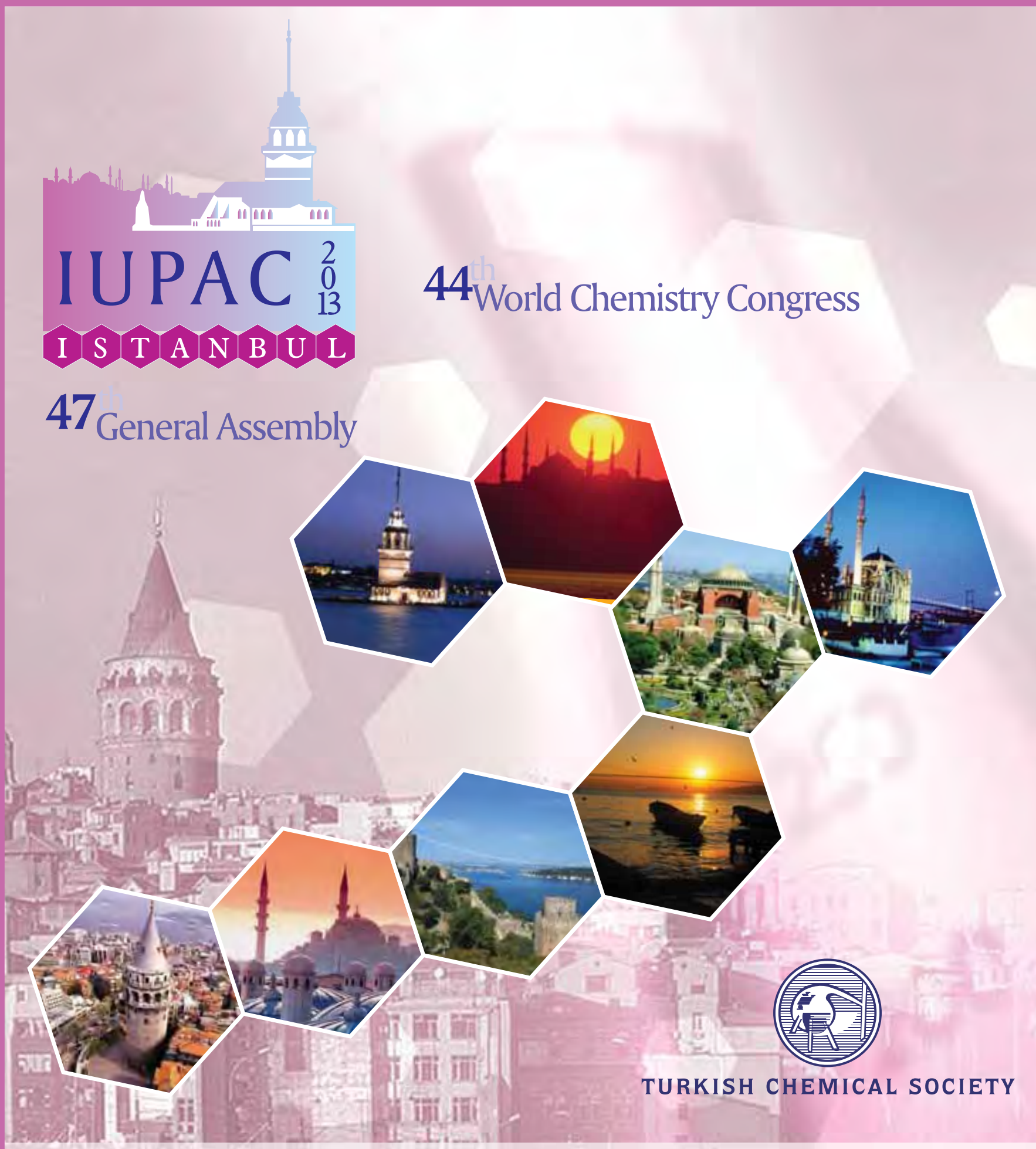

\section{2 - 9 August, 2013 Istanbul, TURKEY}

Erisis

Emin

\section{wWw.iupac2013istanbul.org}

How Wh Hith
Halaskargazi Cad. Tavukcu Fethi Sok. No:28/3 Osmanbey - Sisli - Istanbul / TURKEY Phn:+90 (212) 2966670 pbx Fax:+90 (212) 2966671 bilge.yuksel@brosgroup.net / cem.tuncel@brosgroup.net www.brosgroup.net 